\title{
AVALIAÇÃO DE NITRATO NAS ÁGUAS PROVENIENTES DE FONTES SUBTERRÂNEAS DA BACIA HIDROGRÁFICA DO RIBEIRÃO BORBA GATO, MARINGÁ - PR
}

\author{
D. REZENDE ${ }^{1 *}$, N. C. VALIN JUNIOR ${ }^{2}$, L. NISHI ${ }^{1}$, D. MANTOVANI ${ }^{1}$, A.M. S. VIEIRA ${ }^{3}$, R. \\ BERGAMASCO
}

\author{
${ }^{1}$ Universidade Estadual de Maringá, Departamento de Engenharia Química \\ ${ }^{2}$ Centro Universitário Cesumar, Departamento de Engenharia Ambiental \\ ${ }^{3}$ Universidade Estadual de Maringá, Departamento de Pós Graduação em Engenharia de Alimentos
}

*e-mail: drirezend@gmail.com

\begin{abstract}
RESUMO
O objetivo do presente trabalho foi avaliar a presença de nitrato e verificar o padrão hidroquímico de águas provenientes de fontes subterrâneas da bacia hidrográfica do Ribeirão Borba Gato (BHBG), localizada no município de Maringá - PR. As amostras foram coletadas no primeiro bimestre de 2015 em 19 poços tubulares profundos e encaminhadas ao Laboratório de Gestão, Controle e Preservação Ambiental da Universidade Estadual de Maringá, onde foram submetidas a algumas análises físicoquímicas. Com os resultados laboratoriais foi possível conhecer o padrão hidroquímico da água coletada na área de estudo e, também, verificar possíveis riscos de contaminação antrópica, por meio das concentrações de nitrato. Os resultados demonstram que as águas captadas no aquífero Serra Geral, nesta bacia, possuem influência do aquífero Guarani. A maior parte das amostras (69\%) demonstraram concentrações de nitrato acima dos valores naturais de águas subterrâneas. Assim, é possível verificar que as fontes subterrâneas da BHBG vem sofrendo contaminação, e pode apresentar outros poluentes de igual ou superior periculosidade à saúde humana e ambiental.
\end{abstract}

\section{INTRODUÇÃO}

A qualidade da água subterrânea está relacionada com suas características físicas, químicas e microbiológicas, consequência da capacidade de dissolução de ampla gama de substâncias oriundas do escoamento superficial e subterrâneo que traduz uma série de informações e processos na dinâmica de todo $O$ ecossistema relacionado (DI BERNARDO e PAZ, 2008; LIBÂNIO, 2008).

As características das águas destinadas para o consumo humano são estabelecidas por regulamentações, conforme critérios legais definidos em cada federação. Por isso, a Organização Mundial da Saúde (OMS) recomenda critérios máximos e mínimos para fins potáveis (WHO, 2011). No Brasil estes critérios são estabelecidos pela Portaria do Ministério da Saúde no 2914/2011 (BRASIL, 2011).

Entre os parâmetros de qualidade da água para o consumo humano, o nitrato é o ânion em destaque em várias pesquisas científicas e está relacionada à uma ampla variedade de fontes de contaminação, como industrial, agrícola e urbana. Este composto, presente em concentrações superiores a $45 \mathrm{mg}$ $\mathrm{L}^{-1}$ de $\mathrm{NO}_{3}^{-}$, na água de consumo, está associada à doenças como a metahemoglobinemia e alterações carcinogênicas (WEYER et al., 2001; DE 
ROOS, WARD, CANTOR, 2003; DI BERNARDO e PAZ, 2008; LIBÂNIO, 2008).

Este íon foi constatado sua presença na água subterrânea em níveis superiores ao padrão de potabilidade recomendado pela OMS em aquíferos dos EUA, Bélgica, Espanha, Israel e no Brasil (ORBAN et al., 2010; GODOY et al., 2013; KURTZMAN et al., 2013; LOCKHART, KING, HARTER et al., 2013; RODRIGUEZ-GALIANO et al., 2014).

Os autores relatam que a contaminação está ligada às condições hidrodinâmicas predominantes, locais de mistura, idade da água no aquífero, zona de vulnerabilidade e uso do solo em cada região (industrial, urbano e agrícola)

Neste contexto, o presente trabalho teve como objetivo avaliar a concentração de nitrato, e também, verificar o padrão hidroquímico em águas provenientes de fontes subterrâneas da bacia hidrográfica do Ribeirão Borba Gato (BHBG). A área em estudo está localizada no município de Maringá - PR.

\section{MATERIAIS E MÉTODOS}

A área de estudo é a bacia hidrográfica do Ribeirão Borba Gato (BHBG), localizada no norte do Estado do Paraná, pertencente ao território do município de Maringá.

A BHBG possui área de $22 \mathrm{Km}^{2}$, que, segundo o método de hierarquia fluvial estabelecido por Strahler (1952), utilizado até hoje com êxito, é classificada como bacia de terceira ordem.

A BHBG é uma sub-bacia do rio Ivaí, na qual, a principal fonte de água subterrânea é o Aquífero Serra Geral (ASG) e abaixo, aproximadamente $600 \mathrm{~m}$ de profundidade o Aquífero Guarani (AG).

A Figura 1 ilustra a localização da BHBG no município de Maringá - PR.

Nesta área, conforme plano diretor do município de Maringá (MARINGÁ-PR,
2006) estão presentes diferentes uso e ocupação do solo (urbana, industrial, rural e área de ocupação imediata).

A área de ocupação imediata é caracterizada por loteamentos e pequenas propriedades produtoras de hortaliças; a área rural é predominante a agricultura intensiva (soja, milho, trigo e aveia); na área industrial estão presentes os mais variados ramos, entre galvanoplastia, indústria têxtil, metalúrgicas, lavanderias industriais, alimentícias entre outras; e na área urbana, a região é caracterizada por residências, associações desportivas, hospitais, clínicas e condomínios horizontais.

Figura 1 - Localização da bacia hidrográfica do Ribeirão Borba Gato

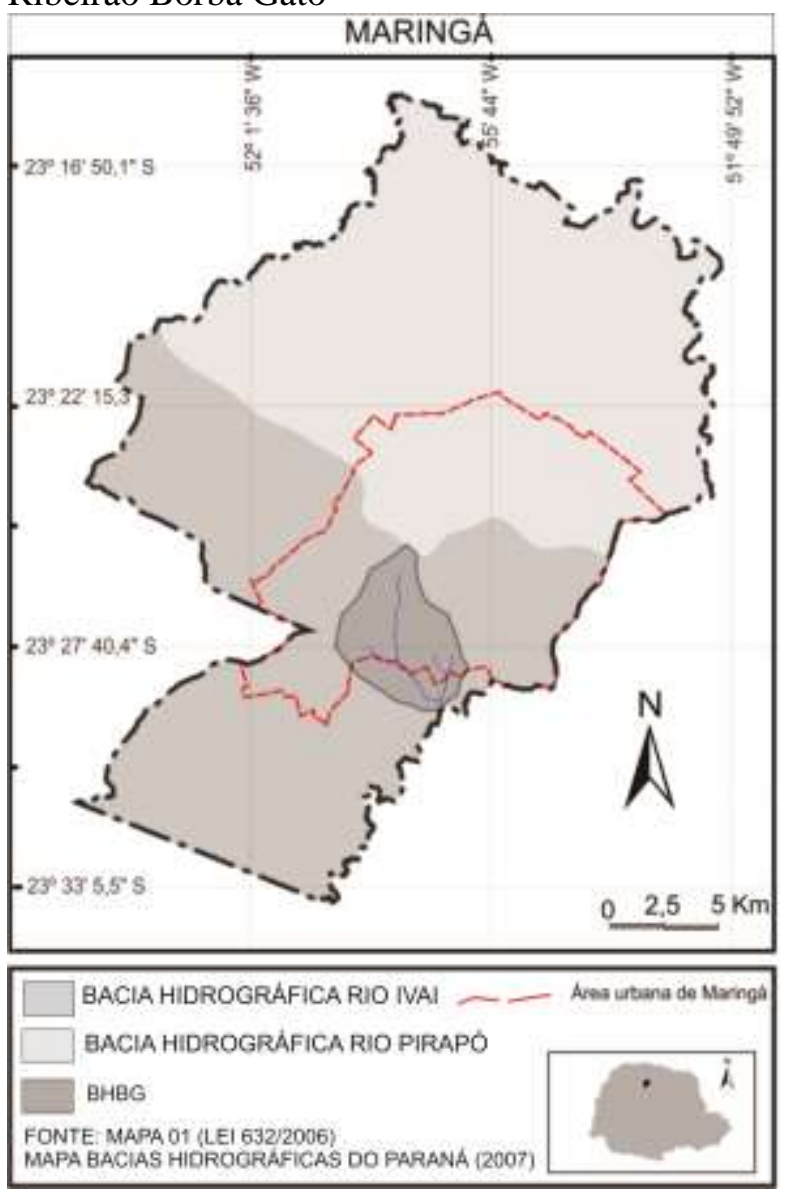

Fonte: Adaptado de Mapa 01 (LEI 632/2006) e mapa Bacias Hidrográficas do Paraná 
A escolha dos poços utilizados para fins potáveis, em estudo, foi realizada de forma aleatória, visando a melhor distribuição possível na área, mediante autorização dos proprietários dos poços, e de acordo com estudos de Lockhart, King, Harter (2013) e Orban et al. (2010).

As coletas foram realizadas no primeiro bimestre de 2015, em 19 poços tubulares profundos, conforme recomendações descritas no "Guia Nacional de Coleta e Preservação de Amostras" (BRANDÃO, 2011). Para a preservação das amostras foi utilizado como referência o Standard Methods for the Examination of Water and Wastewater (APHA, 1998), e encaminhadas ao laboratório de Gestão, Controle e Preservação Ambiental no Departamento de Engenharia Química (DEQ) da Universidade Estadual de Maringá (UEM).

Em laboratório, os parâmetros analisados foram: turbidez (uT), condutividade elétrica $\left(\mu \mathrm{S} \mathrm{cm} \mathrm{cm}^{-1}\right), \mathrm{pH}$, sólidos dissolvidos totais (SDT), bicarbonato, carbonato, cloreto, nitrato, sulfato, sódio, potássio, cálcio e magnésio $\left(\mathrm{mg} \mathrm{L}^{-1}\right)$, de acordo com Standard Methods.

Após analisadas foi possível realizar o cálculo de balanço iônico de acordo com Logan (1965), que define o coeficiente individual de erro das análises, admitindo-se um valor máximo de $10 \%$ para análises aproveitáveis, conforme equação 1 .

$$
\operatorname{Erro}(\%)=\left[\frac{r \sum \hat{a} \text { ioos }+r \sum \text { cátions }}{r \sum \hat{a n i o n s}-r \sum \text { cátions }}\right] x 100
$$

Em seguida, com as amostras que apresentaram erros toleráveis, foi elaborado o gráfico hidroquímico de Piper, com auxílio do software QualiGraf (MOBUS, 2014).

Quanto a avaliação de nitrato, concentrações abaixo de $13 \mathrm{mg} \mathrm{L}^{-1} \mathrm{NO}_{3}^{-} \mathrm{em}$ águas subterrâneas fazem parte do ciclo natural do nitrogênio, enquanto, concentrações acima são indícios de poluição antrópica (NOLAN e STONER, 2000; SHRIMALI e SINGH, 2001). Deste modo, por meio dos resultados de nitrato foi possível verificar se as águas subterrâneas da BHBG estão sendo influenciadas por ações antrópicas ou não.

\section{RESULTADOS E DISCUSSÃO}

Entre as 19 amostras analisados, 6 fontes (P4, P10, P14, P15, P17 e P18) não foram aprovadas no cálculo do balanço iônico, com um coeficiente de erro superior a $10 \%$. Deste modo, os resultados destas fontes não foram utilizados no presente estudo. As Tabelas 2 e 3 apresentam os resultados físicoquímicos de cada amostra.

Tabela 2 - Resultados físico-químicos

\begin{tabular}{lrrrrrrr}
\hline & $\mathbf{N a}^{+}$ & \multicolumn{1}{c}{$\mathbf{K}^{+}$} & $\mathbf{C a}^{+}$ & $\mathbf{M g}^{+}$ & $\mathbf{C l}^{-}$ & $\mathbf{C O}$ & $\mathbf{H C O}_{3}$ \\
\hline P1 & 12,69 & 0,75 & 18,5 & 4,9 & 4,1 & $\mathrm{ND}$ & 113 \\
P2 & 20,85 & 1,06 & 17,25 & 5,5 & 9,5 & $\mathrm{ND}$ & 117,5 \\
P3 & 5,75 & 1,27 & 22 & 7,5 & 10,6 & $\mathrm{ND}$ & 113,5 \\
P5 & 11,65 & 1,155 & 19,615 & 6,7 & 7,1 & $\mathrm{ND}$ & 110,5 \\
P6 & 37,6 & 15 & 12,2 & 3,5 & 7,4 & $\mathrm{ND}$ & 129,5 \\
P7 & 6,85 & 1,365 & 22,61 & 8,3 & 11,7 & $\mathrm{ND}$ & 80,5 \\
P8 & 3,555 & 1,55 & 7,475 & 3,6 & 2,7 & $\mathrm{ND}$ & 56,5 \\
P9 & 92,27 & 0,45 & 5,615 & $\mathrm{ND}$ & 1,4 & 84 & 58,5 \\
P11 & 7,65 & 1,85 & 24,63 & 9,3 & 19,1 & $\mathrm{ND}$ & 125 \\
P12 & 27,85 & 0,6 & 2,6 & 0,5 & 4,1 & 24 & 52 \\
P13 & 13,55 & 0,85 & 23,865 & 3,6 & 4,6 & $\mathrm{ND}$ & 122 \\
P16 & 31,55 & 1,7 & 24,05 & 8,3 & 12,5 & $\mathrm{ND}$ & 169 \\
P19 & 4 & 0,85 & 10,705 & 3,5 & 5,2 & $\mathrm{ND}$ & 36,5 \\
\hline ND: Não detectado & & & & &
\end{tabular}

Tabela 3 - Resultados físico-químicos

\begin{tabular}{lcccccc}
\hline & $\mathbf{S O}^{-}$ & $\mathbf{C E}$ & $\mathbf{p H}$ & Turb & SDT & NO $^{-}$ \\
\hline P1 & 1,2 & 245,05 & 7,6 & 2 & 211 & 10,31 \\
P2 & 1,9 & 288,55 & 6,9 & 0,5 & 291 & 26,84 \\
P3 & 0,5 & 272,4 & 6,8 & 1,5 & 274 & 17,72 \\
P5 & 2,4 & 245,95 & 6,8 & 0,57 & 225 & 17,13 \\
P6 & 1,4 & 315 & 7,4 & 2,2 & 258 & 6,15 \\
P7 & 5,0 & 290,2 & 7,2 & 2 & 310 & 40,8 \\
P8 & 0,2 & 140,85 & 6,3 & 0,56 & 164 & 4,67 \\
\hline
\end{tabular}


Continuação da Tabela 3

\begin{tabular}{lcccccc}
\hline P9 & 0,28 & 411 & 9,5 & 0,74 & 321 & 4,75 \\
P11 & 9,2 & 327,5 & 6,4 & 1,5 & 350 & 19,74 \\
P12 & 0,9 & 286,05 & 9,15 & 0,51 & 220 & 9,21 \\
P13 & 0,4 & 256,4 & 8,0 & 1,5 & 222 & 16,9 \\
P16 & 0,64 & 404 & 6,7 & 1,2 & 290 & 31,8 \\
P19 & 0,3 & 112,25 & 6,4 & 0,8 & 155 & 17,8 \\
\hline
\end{tabular}

Por meio dos dados presentes nas Tabela 2 e 3 foi possível gerar o diagrama de Piper, o qual, forneceu o padrão hidroquímico de cada amostra, conforme Tabela 4.

Tabela 4 - Padrão hidroquímico das amostras em estudo

\begin{tabular}{ll}
\hline P1 & Mista Bicarbonatada \\
P2 & Mista Bicarbonatada \\
P3 & Cálcica Bicarbonatada \\
P5 & Mista Bicarbonatada \\
P6 & Sódica Bicarbonatada \\
P7 & Cálcica Bicarbonatada \\
P8 & Mista Bicarbonatada \\
P9 & Sódica Bicarbonatada \\
P11 & Cálcica Bicarbonatada \\
P12 & Sódica Bicarbonatada \\
P13 & Cálcica Bicarbonatada \\
P16 & Mista Bicarbonatada \\
P19 & Cálcica Bicarbonatada \\
\hline
\end{tabular}

Os resultados presentes nas Tabelas 2, 3 e 4, estão coerentes com outras pesquisas realizadas no ASG (PORTELA-FILHO, FERREIRA, FONSECA, 2002; ROSAFILHO e ROSTIROLLA, 2005; BARROS et al., 2011; ATHAYDE GB, ATHAYDE CVM, ROSA-FILHO, 2012).

Segundo os autores a característica da água presente nas rochas basálticas do ASG é bicarbonatada cálcica, a qual está relacionada com a mineralogia e natureza química dessas rochas.

$\mathrm{Na}$ presente pesquisa, foi constatado a composição mista bicarbonatada e também bicarbonatada sódica, em algumas águas de poços neste ambiente (ASG), o que sugere a mistura com as águas do aquífero subjacente, o Aquífero Guarani (AG). Essa mistura de águas é a consequência de fissuras e descontinuidades rochosas na região da BHBG.

As águas classificadas como cálcica bicarbonatada foram captadas em fontes com entradas de águas em menores profundidades (<60 m), possui $\mathrm{pH}$ entre 6 a 7 e está associada com os maiores valores de nitrato (> $13 \mathrm{mg} \mathrm{L}^{-1}$ de $\mathrm{NO}_{3}^{-}$), indicativo de contaminação antrópica.

As água classificadas como Mista Bicarbonatada foram captadas em fontes com entradas de águas, aproximadamente, entre 60 a 100 metros de profundidades, e possui valores de $\mathrm{pH}$ próximos da neutralidade e variação da concentração de sólidos dissolvidos totais e também de nitrato.

As águas bicarbonatada sódica são característica da influência do AG no ASG, possui $\mathrm{pH}$ acima de 8 e foram coletadas em poços com entradas de águas mais profundas $(>100 \mathrm{~m})$. Apresentaram concentrações naturais de nitrato $\left(<13 \mathrm{mg} \mathrm{L}^{-1} \mathrm{de} \mathrm{NO}_{3}{ }^{-}\right)$.

Em relação ao íon nitrato, foi observado uma grande variação deste, estes valores estão coerentes aos obtidos por Lockhart, King, Harter (2013), que pesquisaram poços e variáveis relacionadas à contaminação de nitrato. Os autores mencionam que a presença de nitrato em poços é característica, em geral, de poços sob influência de efluentes domésticos e efluentes industriais.

Enquanto, Rodriguez-Galiano et al. (2014) mencionam que a contaminação do aquífero está diretamente relacionada com a direção do fluxo das águas subterrâneas e zonas vulneráveis à contaminação.

Contudo, por meio dos resultados apresentados é possível constatar que as águas subterrâneas provenientes da BHBG estão sob influência de contaminação antrópica. Verificado por meio das análises de nitrato em valores acima da concentração natural de águas subterrânea $\left(13 \mathrm{mg} \mathrm{L}^{-1} \mathrm{NO}_{3}^{-}\right)$. 
No entanto, não é possível afirmar a origem da contaminação, pois, como mencionado anteriormente estes parâmetros são indicativos da ação antrópica sob o meio ambiente subterrâneo. Deste modo, a variação dos valores de nitrato, são provenientes dos diferentes usos da BHBG e seu entorno.

\section{CONCLUSÃO}

As águas provenientes de fontes subterrâneas na Bacia Hidrográfica do Ribeirão Borba Gato possuem três classes distintas: Cálcica Bicarbonatada, Mista Bicarbonatada e Sódica Bicarbonatada. Estas águas estão associadas a composição das rochas do ASG e, também, possuem influência das águas do AG.

A maior parte das amostras (69\%) demonstram concentrações de nitrato acima dos valores naturais de água subterrânea. Assim, é possível que as águas subterrâneas da BHBG apresente outros poluentes de igual ou superior periculosidade a saúde humana e ambiental.

Desta maneira, há a necessidade de continuar os estudos na região, e a partir das análises, fornecer resultados conclusivos a respeito da qualidade da água e seus potenciais riscos à saúde da população.

\section{REFERÊNCIAS}

APHA. Standard methods for the examination of water and wastewater, 1998.

ATHAYDE, G. B.; ATHAYDE, C. V. M.; ROSA FILHO, E. F. Compartimentação hidroestrutural e aptidões químicas do Sistema Aquífero Serra Geral no estado do Paraná. Revista Brasileira de Geociências, 42, p. 167-185, 2012.

BARROS, A.; BONGIOLO, S.; SOARES, A. P.; BITTENCOURT, A. V. L.; FERREIRA, F. J. F. Compartimentação estrutural e conectividade hidráulica dos sistemas aquíferos Serra Geral e Guarani: caracterização hidrogeoquímica na região central do Estado do Paraná. Brazilian Journal of Geology, 41(2), p. 319-333, 2011.

BRASIL. Ministério da Saúde. Portaria $n^{\circ}$. 2914, de 12 de dezembro de 2011.

BRANDÃO, C. J. Guia nacional de coleta e preservação de amostras: água, sedimento, comunidades aquáticas e efluentes líquidos.

São Paulo: CETESB, 2011.

DI BERNARDO, L.; PAZ, S. L. P. Seleção de Tecnologias de Tratamento de Água. São Carlos: LDIBETE LTDA, v. 1, p. 878, 2008.

DE ROOS, A. J.; WARD, M. H.; LYNCH, C. F.; CANTOR, K. P. Nitrate in Public Water Supplies and the Risk of Colon and Rectum Cancers. Epidemiology, 14(6), p. 640-649 2003.

GODOY, J. M.; SOUZA, T. A.; GODOY, M. L. D. P.; MOREIRA, I.; CARVALHO, Z. L.; LACERDA, L. D.; FERNANDES, F. C. Groundwater and surface water quality in a coastal bay with negligible fresh groundwater discharge: Arraial do Cabo, Brazil. Marine Chemistry, 156, p. 85-97, 2013.

KURTZMAN, D.; SHAPIRA, R. H.; BARTAL, A.; FINE, P.; RUSSO, D. Nitrate fluxes to groundwater under citrus orchards in a Mediterranean climate: Observations, calibrated models, simulations and agrohydrological conclusions. Journal of contaminant hydrology, 151, p. 93-104 2013.

LIBÂNIO, M. Fundamentos de qualidade e tratamento de água, Átomo, 2008.

LOCKHART, K.; KING, A. M.; HARTER, T. Identifying sources of groundwater nitrate 
contamination in a large alluvial groundwater basin with highly diversified intensive agricultural production. Journal of contaminant hydrology, 151, p. 140-154, 2013.

LOGAN, J. Interpretação de análises químicas d'água. Recife: US. Agency for International Development, 1965.

MARINGÁ-PR, Lei Complementar $\mathbf{n}^{\mathbf{0}}$ 632/2006 - instituiu o Plano Diretor de Maringá, 2006.

MOBUS, G., Qualigraf - Software para auxiliar na análise e interpretação gráfica de dados hidroquímicos. Funceme - Fundação Cearense de Meteorologia e Recursos Hídricos. Disponível para download gratuito em: <http://www.funceme.br/DEHID/qualigra f/index.htm>. Acesso em: 10 out. 2014.

NOLAN, B. T.; STONER, J. D. Nutrients in groundwaters of the conterminous United States, 1992-1995. Environmental Science \& Technology, 34(7), p. 1156-1165, 2000.

ORBAN, P.; BROUYÈRE, S.; BATLLEAGUILAR, J.; COUTURIER, J.; GODERNIAUX, P.; LEROY, M. MALOSZEWSKI, P.; DASSARGUES, A. Regional transport modelling for nitrate trend assessment and forecasting in a chalk aquifer. Journal of contaminant hydrology, 118(1), p. 79-93, 2010.

PORTELA-FILHO, C. V. P.; FERREIRA, F. J.; FONSECA, E. F. R. Estudo preliminar da conexão entre os Aquíferos Serra Geral e Guarani com base em dados aeromagnetométricos e hidroquímicos. In: XII Congresso Brasileiro de Águas Subterrâneas, 2002. Anais... XII Congresso Brasileiro de Águas Subterrâneas Cuiabá, p1-16, 2002.
ROSA-FILHO, F. D. R.; ROSTIROLLA, S. P. Compartimentação magnética-estrutural do Sistema Aquífero Serra Geral e sua conectividade com o Sistema Aquífero Guarani na região central do Arco de Ponta Grossa (Bacia do Paraná). Revista Brasileira de Geociências, 35, p. 369-381, 2005.

RODRIGUEZ-GALIANO, V.; MENDES, M. P.; GARCIA-SOLDADO, M. J.; CHICAOLMO, M.; RIBEIRO, L. Predictive modeling of groundwater nitrate pollution using Random Forest and multisource variables related to intrinsic and specific vulnerability: A case study in an agricultural setting (Southern Spain). Science of The Total Environment, 476, p. 189-206, 2014.

STRAHLER, A. N. Hypsometric analysis of erosional topography. Geological Society of America Bulletin, 63(11), p. 1117-1142, 1952.

SHRIMALI, M.; SINGH, K. P. New methods of nitrate removal from water. Environmental Pollution, 112 (3), p. 351359, 2001.

WEYER, P. J.; CERHAN, J. R.; KROSS, B. C.; HALLBERG, G. R.; KANTAMNENI, J.; BREUER, G.; JONES, M. P.; ZHENG, W.; LYNCH, C. F.; Municipal drinking water nitrate level and cancer risk in older women: the Iowa Women's Health Study. Epidemiology, 12 (3), p. 327-338, 2001.

WORLD HEALTH ORGANIZATION (WHO) Guidelines for drinking-water quality: Fourth edition, 2011.

\section{AGRADECIMENTOS}

Os autores agradecem à CAPES e também à Fundação Araucária, fornecedoras de recursos e bolsas para o desenvolvimento da presente pesquisa. 